\title{
Initiation of meiotic chromosome synapsis at centromeres in budding yeast
}

\author{
Tomomi Tsubouchi, ${ }^{1,3}$ Amy J. MacQueen, ${ }^{1}$ and G. Shirleen Roeder ${ }^{1,2,4}$ \\ ${ }^{1}$ Howard Hughes Medical Institute, Department of Molecular, Cellular and Developmental Biology, Yale University, \\ New Haven, Connecticut 06520, USA; ${ }^{2}$ Department of Genetics, Yale University, New Haven, Connecticut 06520, USA
}

Previous studies of synaptonemal complex assembly in budding yeast have suggested that chromosome synapsis initiates at the sites of crossing over. The data presented here, however, indicate that centromeric regions are preferred sites for synapsis initiation. At early times during meiosis in wild type, the Zip1 protein (a major building block of the synaptonemal complex) localizes specifically to centromeric regions. As synapsis progresses and linear stretches of Zip1 are formed, the majority of stretches are associated with a centromere, as expected if the Zip1 protein present at the centromere polymerized outward along the chromosome arm. In many cases, the centromere is present at one end of a linear stretch, suggesting that synapsis is often unidirectional. Furthermore, the Zip2 protein, a protein that promotes Zip1 polymerization, is often present at the opposite end from the centromere, implying that Zip2 and associated proteins move at the leading edge of Zip1 polymerization. Surprisingly, synapsis initiation at centromeres is independent of the Zip3 protein, which plays a major role in synapsis initiation events at noncentromeric locations. Our data provide evidence for two classes of synapsis initiation events that differ in location, timing, genetic requirements, and relationship to meiotic recombination.

[Keywords: Synaptonemal complex; centromere; meiosis; synapsis initiation; Zip2; Zip1]

Received June 25, 2008; revised version accepted September 24, 2008.

Meiosis is a special type of cell division that produces haploid gametes from diploid parental cells. This reduction in chromosome number is achieved at the first meiotic division, in which homologous chromosomes segregate away from each other, but sister chromatids remain associated. Proper reductional chromosome segregation depends on a complex series of interhomolog interactions that occur during meiotic prophase. An important player in these interactions is the synaptonemal complex (SC), an elaborate proteinaceous structure that holds homologs close together along their lengths (for review, see Page and Hawley 2004). The SC is a tripartite structure consisting of two parallel axial cores, each representing one pair of sister chromatids, and an intervening central region.

In budding yeast, the Zip1 protein serves as the building block of the SC central region (Sym et al. 1993; Sym and Roeder 1995; Dong and Roeder 2000). In the zip1 mutant, axial cores are formed, and these are homologously paired, but not intimately synapsed. Each pair of axial cores is closely connected at multiple sites called axial associations. The formation of axial associations depends on meiotic recombination, suggesting that these

${ }^{3}$ Present address: DNA Recombination Group, Marie Curie Research Institute, Oxted, Surrey RH8 0TL, United Kingdom.

${ }^{4}$ Corresponding author.

E-MAIL shirleen.roeder@yale.edu; FAX (203) 432-3263.

Article is online at http://www.genesdev.org/cgi/doi/10.1101/gad.1709408.

Freely available online through the Genes \& Development Open Access option. junctions mark the sites of recombination events (Rockmill et al. 1995). Furthermore, proteins involved in the initiation of chromosome synapsis localize to axial associations, suggesting that these connections are also the sites where synapsis initiates (Chua and Roeder 1998; Tsubouchi et al. 2006).

A protein assembly referred to as the Synapsis Initiation Complex (SIC) is required for polymerization of Zip1 along the lengths of chromosomes. Components of this complex include Zip2, Zip3, Zip4/Spo22, and Spo16 (Chua and Roeder 1998; Agarwal and Roeder 2000; Tsubouchi et al. 2006; Shinohara et al. 2008). Zip3 acts upstream of the other SIC components and is believed to facilitate and/or stabilize the localization of Zip2, Zip4, and Spo16 to chromosomes. Whereas Zip2 and Zip4 are absolutely required for the formation of $\mathrm{Zip} 1$ linear stretches, a significant amount of SC formation occurs in the absence of Zip3. Here, we show that Zip3 is dispensable for a specific subset of synapsis initiation events.

Several observations have been interpreted to mean that SICs are positioned at the sites of crossing over (for review, see Henderson and Keeney 2005). First, mutations in genes encoding SIC proteins decrease the frequency of crossing over. Second, SICs colocalize and/or physically interact with a number of recombination enzymes. Third, mutations that change the frequency of crossing over lead to corresponding changes in the number of SICs. Finally, SICs, like crossovers, display interference; they are nonrandomly distributed such that two SICs occur close together less often than expected for a 
random distribution. In many respects, SICs resemble the late recombination nodules observed in the electron microscope and believed to mark the sites of crossing over (Carpenter 1988). Thus, it seems likely that SICs and recombination nodules are one and the same, or SICs and recombination nodules are subcomplexes within a larger complex.

In addition to its role as a building block of the SC, Zip1 performs a special role at centromeres (Tsubouchi and Roeder 2005). In mutants defective in the initiation of recombination, and in wild-type cells prior to extensive SC formation, centromeres are joined together in groups of two. This centromere coupling is independent of chromosomal homology and is absolutely dependent on the Zip1 protein, which localizes specifically to centromeres in the absence of recombination initiation. In wild-type cells, most centromere couples initially involve nonhomologous chromosomes, but eventually all centromeres become homologously coupled. It has been proposed that centromere coupling plays a role in homologous chromosome pairing by holding chromosomes together while homology is being assessed (Tsubouchi and Roeder 2005).

Here, we demonstrate another role for Zip1 at centromeres-the Zip1 protein defines centromeres as the earliest sites of synapsis initiation. Indeed, centromeres may be responsible for the bulk of the synapsis that occurs in meiotic cells of budding yeast. The identification of defined sites for synapsis initiation has allowed us to address fundamental questions regarding the mechanisms of synapsis initiation and SC propagation. Our findings bring into question long-standing assumptions regarding the mechanics of SC assembly. We show that chromosome synapsis is often unidirectional, proceeding in only one direction from the site of initiation. Furthermore, we present evidence that SICs are not stationary; instead, these complexes appear to move at the leading edge of Zip1 polymerization.

\section{Results}

\section{Synapsis initiates preferentially at centromeres}

To investigate the association between centromeres and Zip1, we stained surface-spread meiotic nuclei with antibodies to Zip1 and to the centromere component, Ctf19. At early stages of SC formation in wild type, $\sim 75 \%$ of Zip1 dots and short linear stretches are associated with a centromere (Fig. 1A; Tsubouchi and Roeder 2005). To extend this analysis, we selected only those Zipl stretches associated with a single SIC (as opposed to those containing multiple SICs), in order to focus on Zipl entities that most likely represent a single synapsis initiation event. We identified Zipl stretches associated with a single Zip2 focus in nuclei at early zygotene, defined here as nuclei containing 10 or fewer linear stretches of Zipl staining. We found that $81 \%$ of such stretches (123 out of 152; 68 nuclei scored) were associated with a centromere, providing support for the notion that most synapsis initiates at or near centromeres in early zygotene.

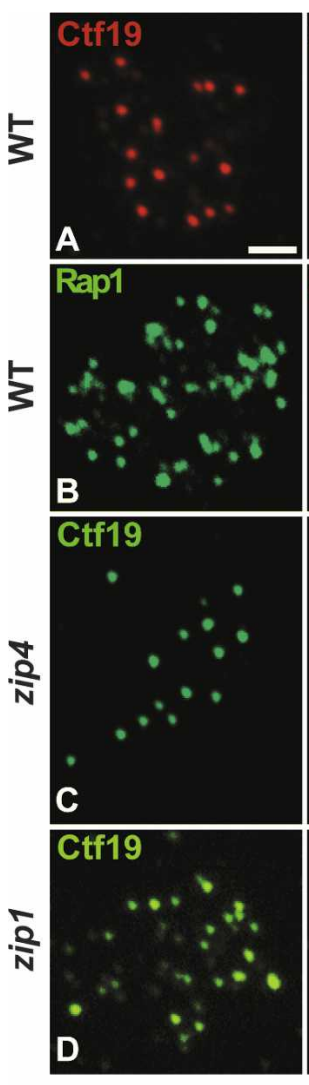

E

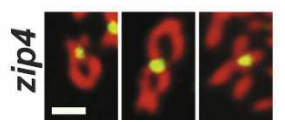

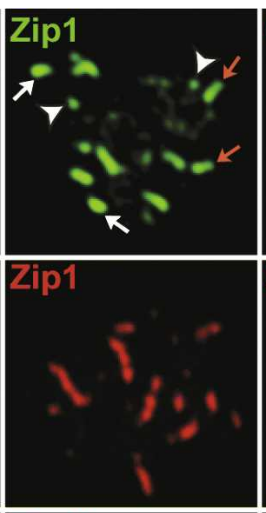
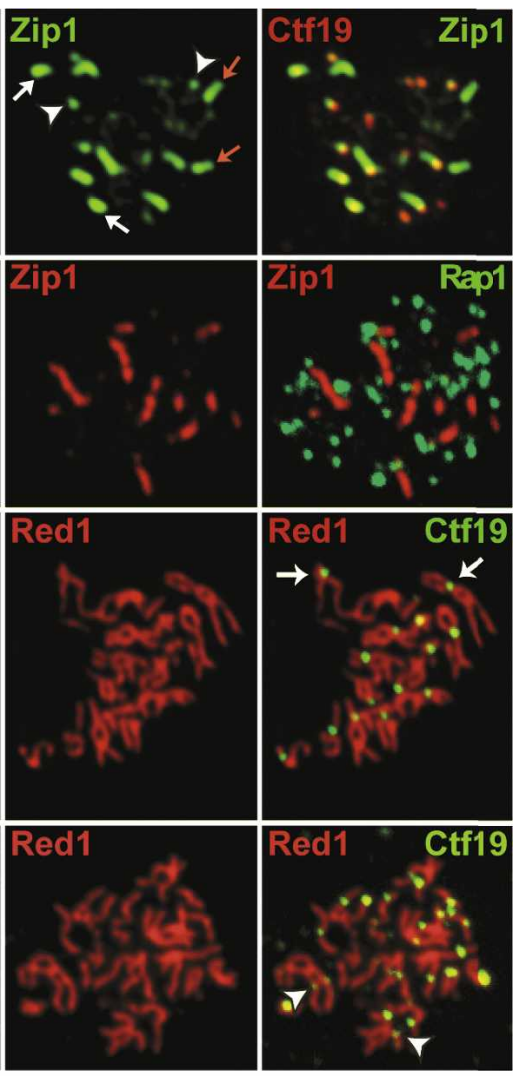

F

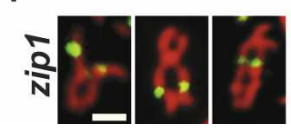

Figure 1. SC formation and homolog associations at centromeric regions. $(A, B)$ Spread nucleus from wild type at early zygotene stained with antibodies to Zip1 and Ctf19 $(A)$ or Zip1 and Rap1 $(B)$. Colors are indicated by the corresponding labels. In $A$, arrowheads indicate Zip1 dots associated with a centromere; white and red arrows indicate Zip1 stretches associated or unassociated with a centromere, respectively. Spread nucleus from zip4 $(C)$ or zip1 $(D)$ stained with antibodies to Ctf19 and Red1. In $C$, arrows indicate centromere foci located at axial associations. In $D$, arrowheads indicate centromere doublets. Bar: $A-D$, $2 \mu \mathrm{m}$. (E) Individual chromosomes from zip4 stained with antibodies to Ctf19 (green) and Red1 (red). (F) Individual chromosomes from zip1 stained with antibodies to Ctf19 (green) and Red1 (red). Bar: E,F, $0.25 \mu \mathrm{m}$

If short chromosomes tend to complete SC formation earlier than other chromosomes, then most or all of the stretches observed at early zygotene might represent fully synapsed chromosomes. Such Zip1 stretches are bound to be associated with centromeres, regardless of where synapsis initiated. The short Zipl stretches present in early zygotene and associated with a single Zip2 focus have an average length of $0.47 \mu \mathrm{m}( \pm 0.2)$. The three smallest yeast chromosomes fall within this size range (based on a total SC length measured at pachytene of 24 $\mu \mathrm{m}$, corresponding to $1 \mu \mathrm{m}$ per $\sim 500 \mathrm{~kb}$ of DNA).

To assess the contribution of fully synapsed chromo- 
somes to the synapsis observed at early zygotene, the ends of chromosomes were visualized by staining with antibodies to the telomeric protein, Rap1 (Fig. 1B). Zip1 stretches that represent fully synapsed chromosomes should have a Rap1 focus at each end. In pachytene nuclei, $86 \%$ (240 out of 279) of linear stretches have a Rap1 focus at both ends, and 14\% (38 out of 279) have a Rap1 focus at only one end (17 nuclei scored). (Note that $6 \%$ [two out of 34] Rap1-free ends are expected even when chromosomes are fully synapsed because the ribosomal DNA does not synapse, thus dividing chromosome XII into two pieces of SC with Rap1 at only one end of each.) In contrast, in early zygotene nuclei, only $18 \%$ (20 out of 109) of Zip1 stretches have a Rapl focus at both ends, and $41 \%$ (45 out of 109) have Rap1 at one end (17 nuclei scored). Thus, most of the Zip1 stretches observed at early times are not simply small chromosomes that are fully synapsed.

\section{Axial associations are found at centromeres}

In the zip1, zip2, and zip4 mutants, the cores of homologous chromosomes are held together at the sites of axial associations (Sym et al. 1993; Chua and Roeder 1998; Tsubouchi et al. 2006). To determine whether axial associations are formed at centromeres, we stained spread chromosomes from the zip4 mutant with antibodies to Ctf19 and to the axial core protein, Red1 (Smith and Roeder 1997). In zip4 cells, only $16( \pm 1)$ centromere foci are observed (25 nuclei scored) (Fig. 1C), indicating that all centromeres are tightly paired and therefore presumably located at axial associations.

In a previous study (Tsubouchi and Roeder 2005), we showed that the centromere coupling observed in a spo11 mutant is absolutely dependent on the Zip1 protein. (There are 16 centromere foci in spo11 and 32 foci in spo11 zip1.) Zip1 might therefore also be responsible for the axial associations formed at centromeres in zip4 cells. Indeed, instead of $16 \mathrm{Ctf} 19$ foci, we observed on average $22( \pm 3)$ Ctf19 foci per nucleus in zip1 cells (25 nuclei scored) (Fig. 1D). The additional foci $(>16)$ almost always appear as doublets (i.e., two foci in close proximity). The doublets are presumed to represent cases where an axial association is not present at the centromere, but homologous centromeres are nevertheless paired by virtue of recombination-dependent axial associations formed elsewhere on the chromosome.

To examine more closely the relationship between axial associations and centromeres in zip4 and zip1 strains, we focused our attention on centromere foci located in regions of spread nuclei where axial cores and axial associations are clearly defined and each Ctf19 focus is clearly associated with a Red1 axis. In the case of zip4, we observed 55 Ctf19 singlets and two doublets. Almost all of the singlets (52 out of 55) were located at an axial association (Fig. 1E). We saw three singlets in which the Ctf19 focus was located in a region where the axes were separate, but a centromere focus was associated with only one axis. These could represent cases in which the axial association was disrupted during the spreading procedure, or they could be staining artefacts (i.e., doublets in which one of two foci was not detected). Thus, at least $90 \%$ (52 out of 57) of centromeres appear to be located at axial associations. The axial associations not located at centromeres are presumed to be connections that depend on recombination.

In the case of the zip1 mutant, of 114 centromeres observed, 84 were present as doublets in which the two foci were located at corresponding positions on homologous chromosomes (Fig. 1F). In addition, we observed 26 Ctf19 singlets located at axial associations and four singlets not located at axial associations. In sum, the centromere appears to be located at an axial association on only $22 \%$ (26 out of 114 ) of chromosomes in zip1. By focusing on regions of spread nuclei where chromosomes are well separated, we see a much higher frequency of doublets, relative to singlets, than by scoring total Ctf19 foci. Even in regions of good spreading, we may underestimate the number of cases in which the centromere is not located precisely at an axial association. Given the small size of yeast chromosomes, and the limited resolution of fluorescence microscopy, there are bound to be cases in which the centromere only "appears" to be located at an axial association. Nevertheless, the fact that a significant fraction of Ctf19 foci are doublets in zip1 (84 out of $114,74 \%$ ), but not in zip4 (only two out of 57 , $4 \%)$, demonstrates that Zip1's role in establishing intimate connections at centromeres (in the absence of SC formation) is not limited to spo11 cells or to wild-type cells prior to recombination initiation.

\section{Zip3 localizes to centromeres in spo11 and in wild-type cells at early zygotene}

If synapsis initiates at centromeric regions, SIC proteins are expected to be present at these sites. Indeed, Zip3 shows robust localization to centromeres, both in the spo11 mutant (which fails to initiate recombination) and in early zygotene nuclei from wild type. In spo11, $18( \pm 2)$ Zip3 foci per nucleus were observed (14 nuclei scored), and $77( \pm 8) \%$ colocalized with centromeres (Fig. 2A), similar to what we reported previously for zip1 (Tsubouchi and Roeder 2005). Of the $15( \pm 1)$ Ctf19 foci detected per nucleus in spo11, $91( \pm 10) \%$ coincided with a Zip3stained focus.

In wild-type cells at very early zygotene, in which Zipl is detected only as dots, $17( \pm 4)$ Zip3 foci were detected (18 nuclei scored), and $67( \pm 16) \%$ colocalized with centromeres (Fig. 2B); furthermore, $69( \pm 16) \%$ of centromere foci colocalized with Zip3. The fact that centromeres are occupied by Zip3 to a lesser extent in wild type than in spo11 presumably reflects the fact that Zip3 has not yet loaded at all centromeres in the very early zygotene nuclei used to analyze wild type.

These data may seem at odds with previous studies indicating that (1) Zip2 and Zip3 colocalize (Agarwal and Roeder 2000), and (2) at pachytene, Zip2 foci are seldom found near centromeres (Fung et al. 2004). We investigated Zip3 localization at centromeres in nuclei with fully synapsed chromosomes by double-staining with an- 
Tsubouchi et al.

Figure 2. SIC components localize to centromeres. Spread nuclei from spo11 $(A)$, wild type at early zygotene $(B)$, and wild type at pachytene $(C)$ stained with antibodies to Ctf19, Zip3, and Zip1. In C, chromosomes indicated by arrowheads are magnified 9.6-fold to show positioning of Ctf19 between Zip3 foci. $(D, E)$ Spread chromosomes from two wild-type nuclei at very early zygotene stained with antibodies to Ctf19, Zip2, and Zip1. The arrows in $D$ indicate foci in which Ctf19 and Zip2 colocalize; the arrowheads in $E$ indicate Ctf19 and Zip2 that lie side by side. Foci indicated by arrows and arrowheads are magnified 12-fold. Bar, $2 \mu \mathrm{m}$.
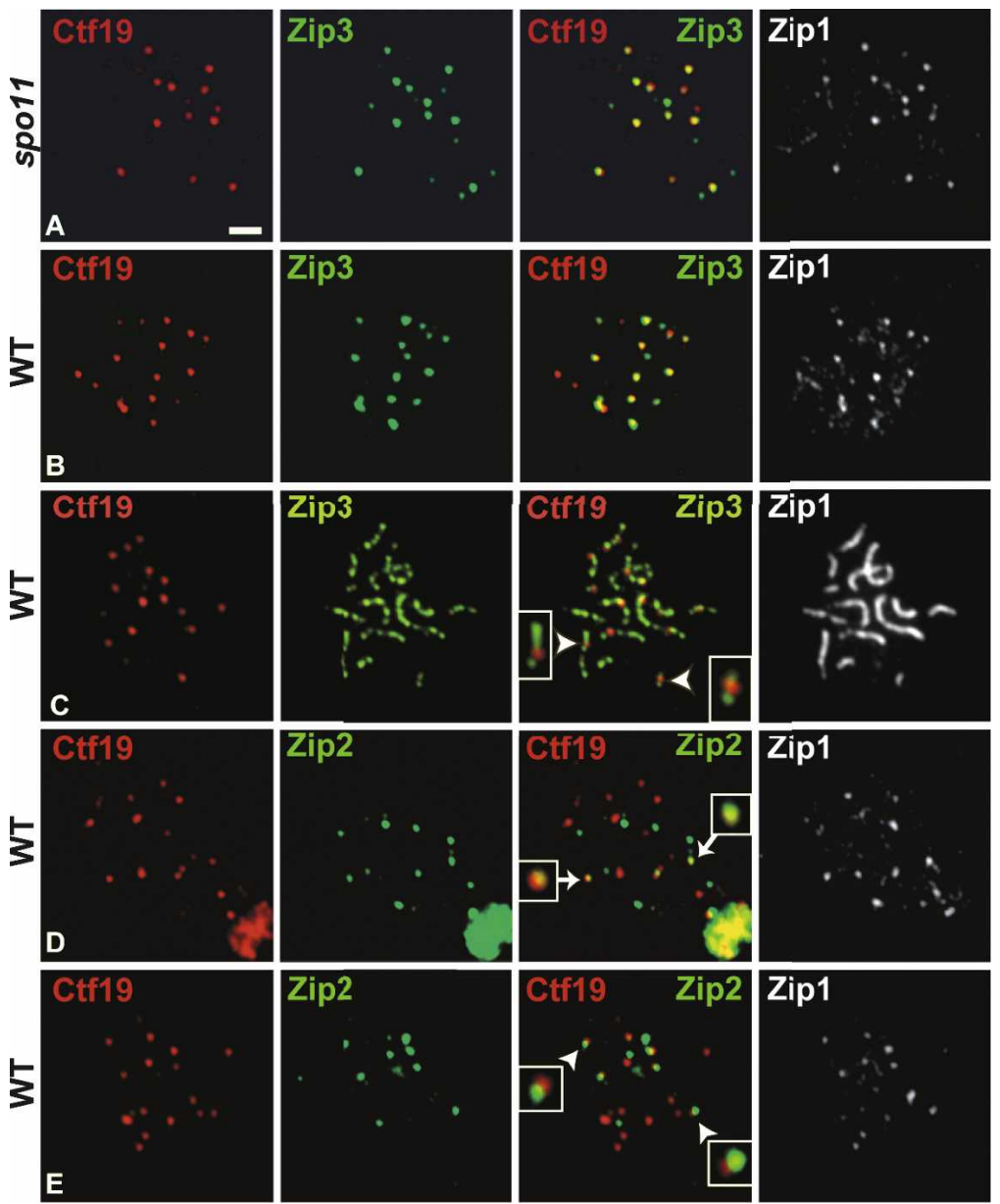

tibodies to Zip3 and Ctf19. At the pachytene stage, when chromosomes are fully condensed and completely synapsed, 50 to $70 \mathrm{Zip} 3$ foci are detected, and these lie immediately adjacent to each other. Yet Ctf19 foci tend not to colocalize with Zip3, but instead are usually located between Zip3 foci (Fig. 2C). The fraction of centromere foci clearly overlapping with Zip3 is only 30\% (105 out of 349), and about one quarter (24 out of 105) of these involve an unusually faint Zip3 focus (22 nuclei scored). Thus, Zip3 localization to centromeres changes as meiosis progresses, starting out very high at zygotene and becoming much lower by pachytene.

\section{Zip2 localizes to centromeres, but to a lesser extent than Zip3}

We failed to detect Zip2 foci in the spo11 mutant, as reported previously (Chua and Roeder 1998). Thus, unlike Zip3, the localization of Zip2 to centromeres appears to depend on recombination initiation or another Spol1-dependent process.

In wild-type nuclei at very early zygotene, fewer Zip2 foci were present on chromosomes (on average $9 \pm 5$ foci), compared with Zip3, and the number of Zip2 foci varied considerably from one nucleus to another. The rate of colocalization between Zip2 and centromeres also showed wide variation; in sum, 16\% (38 out of 236) of Zip2 foci colocalized with centromeres (Fig. 2D). Interestingly, Zip2 foci were often found immediately adjacent to centromeres (center-to-center distance between foci of $0.21 \pm 0.07 \mu \mathrm{m}$ ) (Fig. 2E); $22 \%$ (52 out of 236) of Zip2 foci were in this category. Thus, a total of $38 \%$ of Zip2 foci were either at or immediately adjacent to centromeres. The frequency of both Zip2/Ctf19 colocalization and side-by-side localization is significantly greater than expected by chance $\left(P=1.1 \times 10^{-8}\right.$ for colocalization, and $P=2.1 \times 10^{-5}$ for side-by-side localization) (see the Materials and Methods).

\section{Evidence for unidirectional chromosome synapsis and SIC movement}

In an effort to understand how SC assembly normally proceeds from an initiation site, we focused our attention on centromere-associated Zip1 stretches present at early zygotene. We identified centromere-associated, linear Zip1 stretches with either one or two Zip2 foci and then examined the location(s) of Zip2 relative to the centromere. Of 165 such stretches examined, 84 contained a single Zip2 focus (Figs. 3, 4). Strikingly, in half of these, the Zip2 focus was present at one end of the Zip1 stretch, and the centromere was located at the opposite end (Figs. 
A
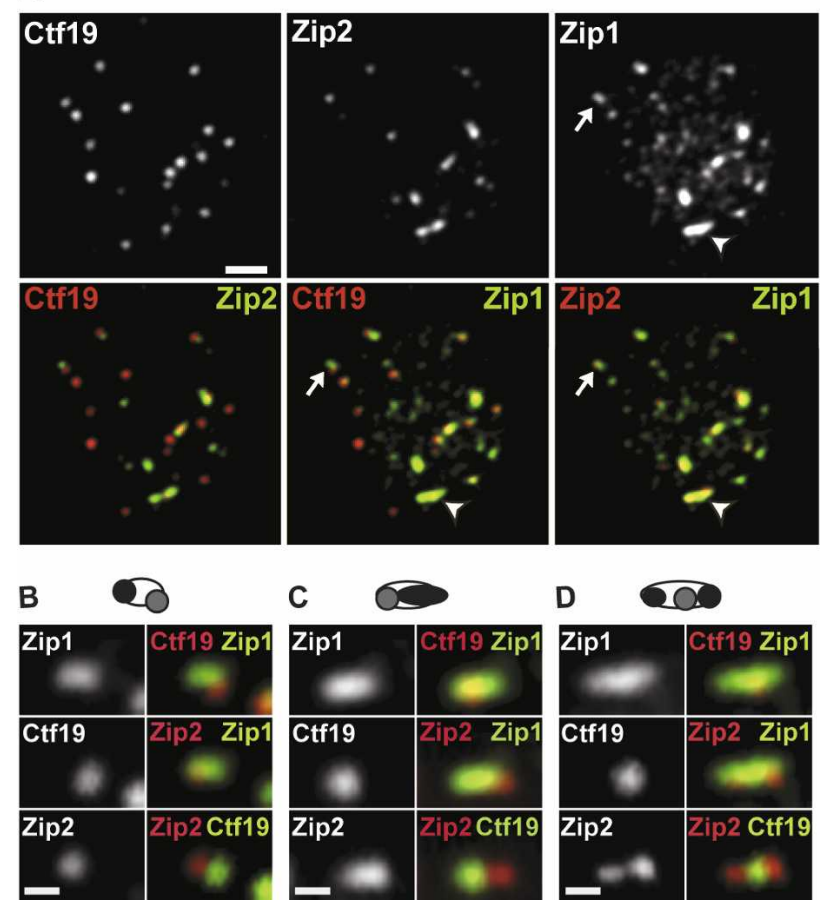

Figure 3. Zip2 and centromere configurations in short, linear Zip1 stretches. (A) Spread nucleus from wild type at early zygotene stained with antibodies to Ctf19, Zip2, and Zip1. The arrows and arrowheads indicate Zip1 stretches containing one or two Zip2 foci, respectively. Bar, $2 \mu \mathrm{m}$. $(B-D)$ Examples of the most common configurations of Zip2 and Ctf19. In the diagram at the top of each panel, unfilled ovals indicate Zip1; gray circles indicate centromeres, and black regions indicate Zip2. Bar, $0.125 \mu \mathrm{m}$.

$3 B ; 4 a)$. In several of these (15 out of 42 ), Zip2 staining was elongated along the axis of Zip1 staining, with Zip2 most concentrated at the centromere-distal end of the stretch but trailing back toward the centromere (Fig. 3C). There were several other categories of Zip1 stretches, but the number in each category was relatively small (Fig. 4). Three categories (Fig. 4b,d,e) were similar to the major group in that Zip2 and the centromere were located at some distance from each other; however, Zip2 and/or the centromere were not located at the extreme end of the Zip1 stretch (see also legend to Fig. 4). Other categories (Fig. 4c,f) were clearly different from the predominant class in that the Zip2 focus coincided with the centromere. Intriguingly, three quarters of the Zip1 stretches with a single Zip2 focus had Zip2 located at one end of the stretch.

We also analyzed 81 short Zip1 stretches associated with two Zip2 foci (Fig. 4). In the predominant class (Fig. $4 \mathrm{~g}$ ), the Zip1 stretch was flanked on both sides by a Zip2 focus with the centromere located somewhere in between (Fig. 3D). In the second major category (Fig. 4h), the two Zip2 foci were located on the same side of the centromere. In the third most frequent class (Fig. 4i), Zip2 was located at one end of the stretch, while Zip2 and the centromere were colocalized at the other end.
In the centromere-associated Zip1 stretches present at early times, the pattern of Zip3 staining is somewhat different from that of Zip2 (Fig. 4). We found more Zip1 stretches with two Zip3 foci as opposed to those with one. The most common class exhibited a Zip3 focus at one end with both Zip3 and the centromere colocalized at the other end. The second most common configuration was one in which Zip3 was present at both ends of the Zipl stretch and the centromere was located somewhere in between.

The fact that SIC proteins are often located at the end of a Zip1 stretch opposite to the centromere, sometimes trailing back toward the centromere, raises the possibility that SIC proteins move along chromosomes as syn-

\begin{tabular}{|c|c|c|}
\hline One SIC & Zip2 & Zip3 \\
\hline$a \bigcirc$ & 42 & 8 \\
\hline b 0 & 11 & \\
\hline c 0 & 10 & \\
\hline d 0 & 9 & \\
\hline e $\infty$ & 8 & 4 \\
\hline$f 0$ & 4 & \\
\hline Total & 84 & 12 \\
\hline Two SICs & $\underline{\text { Zip2 }}$ & $\underline{\text { Zip3 }}$ \\
\hline 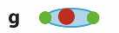 & 39 & 10 \\
\hline h 0 & 11 & 9 \\
\hline i $\square$ & 9 & 15 \\
\hline j $\infty$ & 7 & 2 \\
\hline$k 00$ & 4 & \\
\hline 10 & 3 & 1 \\
\hline$m \infty$ & 3 & \\
\hline $\mathbf{n} \circlearrowright$ & 3 & 1 \\
\hline - 00 & 2 & \\
\hline Total & 81 & 40 \\
\hline
\end{tabular}

Figure 4. Categories of SIC and centromere configurations in short, linear Zip1 stretches. The different patterns of staining observed in Zip1 stretches containing one or two Zip2 or Zip3 foci are diagrammed, with the numbers observed indicated to the right of each category. Zip1 is indicated in blue, Ctf19/ centromeres are in red, and Zip2 or Zip3 are in green. Zip1 stretches in category $a$ differed in the degree of overlap between Zip1 and the Ctf19 focus: At one extreme, Zip1 and the Ctf19 focus overlapped only slightly, while at the other extreme, Zip1 staining extended all the way to the outside end of the Ctf19 focus. The same was true for the overlap between Zip1 and the Zip2 or Zip3 focus. It seems likely that these differences are artifacts of the spreading procedure; a Ctf19/Zip2/Zip3 focus located at one end of a Zip1 stretch may land in slightly different places relative to the Zip1 stretch when the three-dimensional object becomes compressed into two-dimensional space. In cases where Zip1 staining extended beyond the region of Ctf19 staining (a little or a lot), and Zip2 was located at the opposite end, then the stretch was placed in category $b$. Thus, some members of category $b$ were difficult to distinguish from those in category $a$. Similarly, in category $d$, there was variation in the distance of the Zip2 focus from the end of the Zip1 stretch, and some stretches were only subtly different from those in category $a$. Similar logic and concerns apply to the assignment of Zip1 stretches in the other categories listed. For Zip2/Ctf19 localization, 44 nuclei were scored; for Zip3/Ctf19 localization, 19 nuclei were scored. 
Tsubouchi et al.

apsis progresses (see the Discussion). This notion can explain why centromeric localization of SIC proteins is high in early zygotene, but very low at pachytene.

\section{Synapsis initiation at centromeres is largely} independent of Zip3

The data presented here, in conjunction with results published previously, argue that synapsis initiates both at centromeres and at noncentromeric locations, with centromere-associated synapsis predominating at early times. Do these two types of synapsis initiation differ mechanistically? One significant difference is that Zip1 is required for Zip3 localization to centromeres, but not to noncentromeric locations. In the zip4 mutant, the number of Zip3 foci on meiotic chromosomes is $26( \pm 5)$, and $15( \pm 2)$ of these colocalize with centromeres (25 nuclei scored) (Fig. 5A). In contrast, in zip1, the number of Zip3 foci is reduced to $15( \pm 5)$, and only $2( \pm 1)$ of these are located at centromeres (25 nuclei scored) (Fig. 5B).

Curiously, in zip3 mutant cells, SC formation initiates predominantly from centromeres. We examined the association between centromeres and Zip1 by scoring short Zip1 stretches (0.35-0.65 $\mu \mathrm{m}$ in length) in a population of nuclei at different stages of meiotic prophase. (Note that this differs from the analysis reported above, where we examined short stretches only in nuclei at an early stage of synapsis.) About half (152 out of 282; 54\%) of the short Zipl stretches in wild-type nuclei were associated with a centromere (151 nuclei scored), whereas a much greater fraction (247 out of 290; 85\%) were centromere-associated in the zip3 mutant (210 nuclei scored). This statistically significant difference $(P<0.0001)$ indicates that centromeric regions are less dependent on the Zip3 protein for synapsis initiation. Fluorescent in situ hybridization, probing the centro- mere of chromosome IV, indicates that SC is assembled between homologous chromosomes in the zip3 mutant (data not shown).

Additional evidence that synapsis initiates predominantly at centromeres in the zip3 mutant comes from analysis of the total number of Zip1 linear stretches (Fig. 6). In zip3, only two out of 150 nuclei examined exhibited more than $16 \mathrm{Zip} 1$ stretches (16 is the number of chromosome pairs in yeast). In contrast, in wild type, a significant fraction of cells displayed $>16$, and as many as 23, distinct Zip1 stretches.

\section{Discussion}

\section{Synapsis initiates at centromeres}

The observations presented here argue that centromeres are preferred sites for synapsis initation during early stages of SC formation in budding yeast. In zygotene nuclei, the majority of Zip1 linear stretches are associated with centromeres. Staining with antibodies to Rap1 to identify telomeres demonstrates that most of these short stretches are not simply short chromosomes that have completed synapsis. Furthermore, SIC components localize to centromeres, and axial associations are located at centromeres. Unlike synapsis initiation at noncentromeric locations, SC formation initiating at centromeres is largely independent of the Zip3 protein, suggesting that these two types of synapsis initiation are mechanistically distinct.

Do the short Zip1 stretches present in early zygotene nuclei represent SC formation between homologous chromosomes? In principle, Zip1 might polymerize between nonhomologous chromosomes, then depolymerize to allow the mismatched chromosomes to dissociate. In our previous study (Tsubouchi and Roeder 2005), we

\section{A zip4}
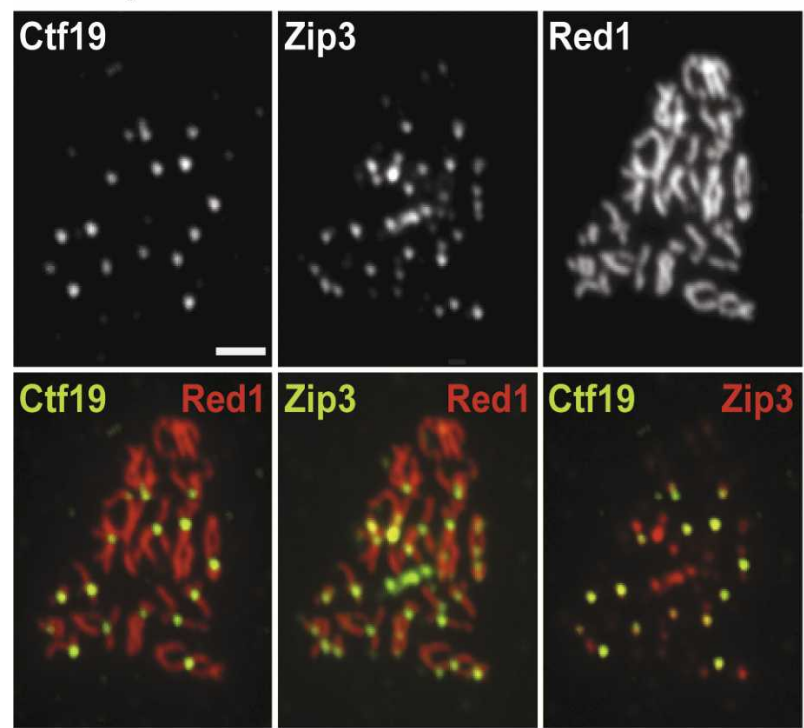

\section{B $\quad$ zip1}
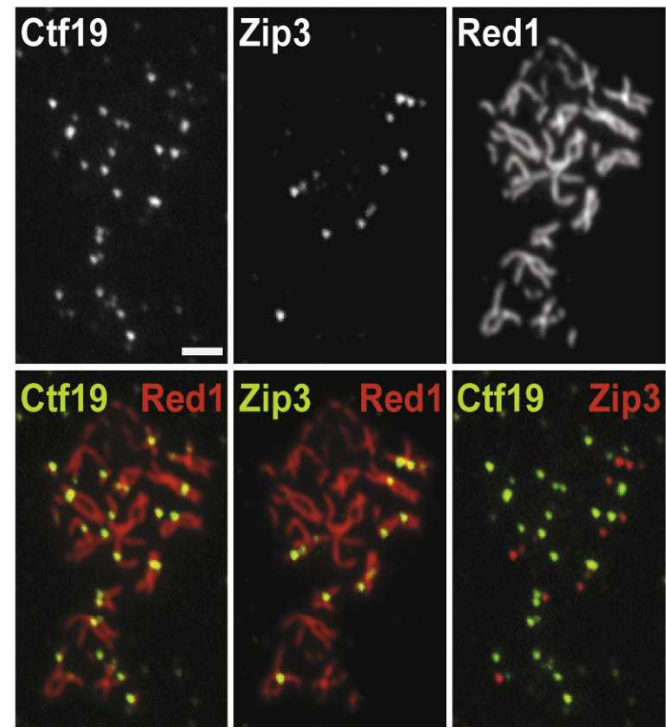

Figure 5. Zip3 localization in mutants defective in SC formation. Spread nucleus from zip4 $(A)$ or zip1 $(B)$ stained with antibodies to Ctf19, Zip3, and Red1. Bar, $2 \mu \mathrm{m}$. 


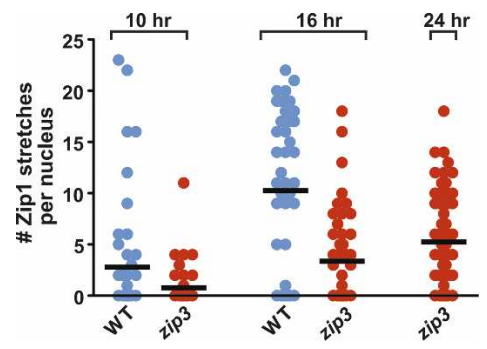

Figure 6. Number of Zip1 stretches in wild type and zip3. The number of Zip1 stretches in nuclei from wild type after 10 and $16 \mathrm{~h}$ of sporulation medium, and zip3 after 10, 16, or $24 \mathrm{~h}$ of sporulation, is displayed on a scatterplot. A later time point was examined for zip3 because synapsis is delayed in this mutant, compared with wild type. Each nucleus is plotted along the $Y$-axis according to the total number of distinct Zip1 stretches it contained. A horizontal black bar depicts the mean for each strain.

used centromeres tagged with lacO repeats bound to LacI-green fluorescent protein to assess homologous pairing. In early zygotene nuclei, all of the nuclei in which a Zip1 linear stretch was associated with the centromere of chromosome XI contained a single centromere XI focus (T. Tsubouchi and G.S. Roeder, unpubl.), arguing that Zip1 polymerization is restricted to homologously paired chromosomes. Furthermore, even in nuclei in which Zip1 had not begun to polymerize along chromosome XI, the frequency of centromere XI pairing was $\sim 50 \%$.

Based on our previous report (Tsubouchi and Roeder 2005) that short Zip1 stretches are associated with centromeres, Henderson and Keeney (2005) have argued that centromeres serve as barriers to SC formation, rather than sites of initiation. While we cannot formally exclude the possibility that synapsis initiates elsewhere and terminates at the centromere, our observations favor the view that synapsis starts at centromeres. Even at very early stages of synapsis, when Zip1 is present only as dots on chromosomes, these foci are preferentially associated with centromeres. This recombination-independent localization of Zip1 may give centromeres a head start over other chromosomal sites when signals downstream from recombination initiation trigger SC assembly. Furthermore, if centromeres do serve as SC termination sites, then the only way to account for our observations is to presume that synapsis initiates preferentially in centromere-proximal regions and then proceeds specifically in the direction of the centromere. However, we do not observe a high frequency of short Zip1 stretches lying close to a centromere, but not overlapping with the centromere (data not shown), which is the predicted intermediate in synapsis initiating elsewhere and proceeding toward the centromere.

\section{Roles for Zip2 and Zip3 at centromeres}

If synapsis initiates at centromeres, then protein components of SICs should be found at these locations. Indeed, we do find that Zip2 and Zip3 localize to centromeres in zygotene nuclei. Paradoxically, however, we find that
Zip3 is not required for SC formation initiating at centromeres. Indeed, unpublished data (A.J. MacQueen and G.S. Roeder, unpubl.) indicate that Zip3 functions specifically at centromeres to prevent synapsis in the absence of recombination. Do these observations preclude a role for Zip3 in promoting synapsis at centromeres? We note that chromosomal localization of Zip2 is strongly dependent on Zip3, even at centromeres (Tsubouchi et al. 2006). Furthermore, the rate of chromosome synapsis in zip3 is delayed compared with wild type (Agarwal and Roeder 2000; A.J. MacQueen and G.S. Roeder, unpubl.). Thus, Zip3 might function both to prevent synapsis initiation at centromeres prior to recombination initiation and then to enhance initiation at centromeres subsequent to recombination initiation by helping to recruit Zip2.

Zip2 is essential for synapsis initiation, regardless of where it occurs on the chromosome, raising the question: Why is the rate of Zip2 colocalization to centromeres lower than that of Zip3? Recall that Zip3 localization to centromeres is independent of Spo11, but Zip2 localization depends on Spo11. Zip3's head start in localizing to centromeres might account for the measured difference between the centromere colocalization rates of these two SIC components. Alternatively, or in addition, perhaps Zip2 moves away from the centromere as soon Zip1 polymerizes, whereas some or all of the Zip3 protein remains transiently behind (see below). Note that essentially all $(99 \%)$ of the short Zip1 stretches present in early zygotene nuclei are associated with at least one Zip2 focus.

\section{Do all SICs promote SC formation?}

At the pachytene stage, the number of SICs per nucleus is $\sim 60$. If all SICs contribute equally to synapsis, then centromere-derived SICs should account for approximately one quarter of the SC that is formed. However, a couple of observations suggest that centromeric SICs contribute more than their "fair share" to Zip1 polymerization. First, in early zygotene nuclei, most synapsis initiates at centromeres, so a significant amount of synapsis has already occurred before most noncentromeric SICs come into play. Second, in wild-type cells, we observe a maximum of $\sim 23$ distinct Zip1 stretches, not the 60 that might be expected if all SICs are active. Clearly, counts of Zip1 stretches can underestimate the number of independently initiated synapsis events because (1) Zip1 stretches initiating at adjacent SICs may quickly fuse to create a single stretch, and (2) separate stretches that lie close together may appear as one at the level of resolution of fluorescence microscopy. Nonetheless, our data raise the possibility that only a subset of the SICs observed cytologically actually function to trigger Zip1 polymerization de novo. The remaining SICs may nevertheless play a role in crossing over.

\section{Evidence for unidirectional chromosome synapsis}

Our studies of synapsis initiation at centromeres reveal two unexpected features of SC formation. The fact that 
the centromere is often found at one end of nascent Zip1 stretches suggests that synapsis frequently occurs unidirectionally, starting at the centromere and proceeding outward in a single direction. It is possible that all synapsis initiation events at centromeres proceed in a single direction; in this case, Zip1 stretches with a centromere in the middle would represent two distinct firing events, both occurring at the centromere but proceeding outward in opposite directions.

The finding of unidirectional synapsis contrasts with the long-standing assumption that SC polymerization proceeds outward in both directions from synapsis initiation sites (e.g., see King and Mortimer 1990; Roeder 1997; Henderson and Keeney 2005; Joyce and McKim 2007). However, SC stretches with a nodule (i.e., SIC) at one end have been observed in electron microscopic studies of Sordaria (Zickler et al. 1992), suggesting that this phenomenon is not unique to budding yeast.

\section{SICs move as synapsis progresses}

Another surprising feature of our data is that SICs appear to move as synapsis progresses. SIC proteins start out at the centromere, but are found at some distance from the centromere as the Zip1 stretch elongates. SIC movement can account for the fact that centromeric regions show a low density of SICs (relative to other chromosomal regions) at pachytene (Fung et al. 2004), even though SICs are found preferentially at or near centromeres in early zygotene.

In our analysis of short Zip1 stretches containing a single Zip2 focus, we found that the centromere and Zip2 are most often located at opposite ends of the Zip1 stretch. Assuming that SC formation initiates at centromeres in these cases, the simplest explanation for Zip2 being at the opposite end is that Zip2 moves away from the centromere as Zip1 polymerizes. Consistent with this notion, we found that Zip2 is often elongated along the axis of the Zip1 linear stretch, with staining most intense at the centromere-distal end of the stretch but trailing back toward the centromere. These observations raise the intriguing possibility that the bulk of Zip2 is located at the leading edge of Zip1 polymerization and moves along chromosomes as synapsis progresses.

We also analyzed short Zip1 stretches associated with two Zip2 foci. In approximately half of these, the Zip1 stretch was flanked on both sides by a Zip2 focus with the centromere located somewhere in between. This configuration is expected from bidirectional synapsis, simultaneously proceeding outward in both directions from the centromere. Alternatively, this configuration could result from two synapsis initiation events at the centromere, each triggering Zip1 polymerization in a different direction.

The pattern of Zip3 staining is different from that of Zip2 at early times. Many of the short Zip1 stretches have two Zip3 foci, with Zip3 at one end and Zip3 and Ctf19 colocalized at the opposite end. Thus, some of the Zip3 protein appears to remain at the centromere (or become reloaded) after Zip2 has departed, perhaps to aid in recruiting Zip2 and thus facilitating a second initiation event. Consistent with this possibility, we also observed several examples of Zip1 stretches with Zip3 at both ends and the centromere in the middle, as expected from two rounds of synapsis initiation proceeding in opposite directions from the centromere.

Some of the centromere-associated Zip1 stretches examined cannot be explained by synapsis initiating at the centromere and proceeding outward in one or both directions (Fig. 4b-f,h,j,k-o). However, it should be noted that, in most of the minor categories, there were some stretches that were difficult to distinguish from those in the major classes (see the legend for Fig. 4); thus, the absolute number in each category is uncertain. Nevertheless, at least some of the stretches appear to be due to synapsis initiation at noncentromeric locations. A more detailed interpretation is not possible since it is not known whether noncentromeric SICs move along chromosomes as synapsis progresses, nor is it known whether synapsis initiating at noncentromeric SICs is unidirectional or bidirectional.

Cheng et al. (2006) found that the Zip3 protein is a SUMO E3 ligase that modifies proteins present in the cores of meiotic chromosomes; they further found that the Zip1 protein binds to SUMO-conjugated products. They therefore proposed that Zip3 moves along chromosomes, modifying chromosomal cores in order to facilitate Zip1 polymerization. Hooker and Roeder (2006) also proposed that sumoylation occurs at the leading edge of Zip1 polymerization based on their observations that localization of SUMO along chromosomes depends on Zip1 polymerization, and synapsis is impaired by a mutation in the UBC9 gene (which encodes a SUMO-conjugating enzyme). If SUMO modification of chromosome cores is required for synapsis, then how can synapsis initiating at the centromere proceed in the absence of Zip3? Cheng et al. (2006) found that at least two different sumoylation activities are active during meiosis. In early zygotene, SUMO is found at centromeres, and this localization is Zip3-independent. As synapsis progresses, Zip3's modification activity is up-regulated, while other SUMO E3 ligases are down-regulated. These other SUMO ligases may account for the early, centromereassociated Zip1 stretches.

\section{Do the SICs present at pachytene mark the sites of crossovers?}

Previous studies suggested that the SICs observed at pachytene mark the sites of crossing over (see above). How can this notion be reconciled with our observation that the SICs present in early zygotene are located preferentially near centromeres, even though meiotic crossing over is repressed in the vicinity of the centromere (Lambie and Roeder 1986)? One possibility is that the SICs assembled at centromeres move along chromosomes until they encounter a site committed to crossing over and then remain at that location. Thus, even SICs originating at centromeres would eventually be located at crossover sites. Another possibility is that centro- 
mere-derived SICs do not mark crossover sites, but other SICs are associated with crossovers. This might explain why there is not perfect colocalization between the Zip2/Zip3/Zip4 proteins and gene products believed to be directly involved in crossing over, such as Msh4 and Msh5 (Novak et al. 2001). Imperfect colocalization could also be explained by differences in the timing of localization of different proteins and/or to difficulties in detection.

Fung et al. (2004) observed interference between SICs in both wild type and the zip1 mutant. Movement of SICs during synapsis would tend to randomize SIC distribution, so how can SICs display interference both in the absence of synapsis and after synapsis has occurred? This finding can be explained if (1) most SICs do not initiate synapsis and therefore do not move, and (2) most of the SICs that do move are not associated with crossing over (i.e., are centromeric SICs).

\section{Specialized sites for synapsis initiation in other organisms}

Yeast is not the only organism that uses specialized sites for synapsis initiation. For example, in the human male, synapsis initiates preferentially in subtelomeric regions (Brown et al. 2005). In Caenorhabditis elegans, synapsis appears to initiate almost exclusively at a single site per chromosome, called the Pairing Center (MacQueen et al. 2005). Synapsis in yeast and worms has been assumed to be very different mechanistically because synapsis in yeast depends on recombination, whereas synapsis in worms can occur in the absence of recombination initiation. However, our observations that yeast centromeres serve as defined sites of synapsis initiation, and that the initial localization of Zip1 to these sites is independent of recombination, argue that yeast is more like worms than has generally been assumed. A curious feature of synapsis initiation events at centromeres is that, while synapsis does not (presumably) initiate at the site of a recombination event, the initiation of recombination (i.e., Spo11 function) is required for Zip1 elongation to occur. This observation suggests some sort of signaling whereby recombination initiation at other chromosomal locations allows Zip2 to localize to centromeres and promote synapsis between homologously paired chromosomes. This coupling between recombination initiation and chromosome synapsis may provide a means to ensure that synapsis takes place specifically between homologous chromosomes.

\section{Materials and methods}

\section{Yeast strains}

All strains are diploids in which both haploid parents are isogenic with BR1919-8B (Rockmill et al. 1995). Diploids were made by mating appropriate haploids. Haploids were generated by transformation and/or genetic crosses. All strains are homozygous for the markers indicated.

Plasmids for introducing the following gene disruptions or gene tagging were described previously: zip 1::LEU2 (Sym et al. 1993), zip4::ADE2 (Tsubouchi et al. 2006), spo11::ADE2 (Engebrecht and Roeder 1989), zip3::URA3 (Agarwal and Roeder 2000), CTF19-MYC-KAN (Tsubouchi and Roeder 2005), ZIP3GFP-URA3 (Agarwal and Roeder 2000) and ZIP2-GFP@URA3 (Chua and Roeder 1998).

\section{Cytological analysis}

Meiotic chromosome spreads, staining, and imaging were carried out as described by Tsubouchi et al. (2006). The following primary antibodies were used: rabbit anti-Zip1 antibodies at 1:100 dilution (Rockmill et al. 2003), rabbit anti-Red1 at 1:400 dilution (Rockmill et al. 2003), rabbit anti-Rap1 at 1:200 dilution (Pardo and Marcand 2005), guinea pig anti-GFP at 1:100 dilution (Agarwal and Roeder 2000), and mouse anti-myc (Covance) at 1:100 dilution. The following secondary antibodies were obtained from Jackson Immunoresearch and used at 1:200 dilution: goat anti-rabbit FITC, goat anti-rabbit TxRed, donkey anti-mouse CY5, donkey anti-mouse TxRed, donkey antimouse CY3, donkey anti-guinea pig TxRed, and donkey antiguinea pig FITC. DAPI was used at $1.5 \mu \mathrm{g} / \mathrm{mL}$.

Throughout the text, "Zip1 stretches" refer to those regions of Zip1 staining in which the length to width ratio was at least 1.75 to 1 . The lengths and widths of Zip1 stretches were measured using IPLab Spectrum software (Scanalytics, Inc.) or the Measure Distance tool in the Softworx deconvolution software (Applied Precision).

To test whether colocalization and side-by-side localization between Zip2 and centromeres can occur by chance at the rates observed, images were rotated $90^{\circ}$ to see if $\mathrm{Zip} 2$ and $\mathrm{Ctf} 19$ foci colocalized or were located side-by-side at similar rates before and after turning within the selected circular DAPI-stained area. After rotation, only $4.3 \%$ of Zip2 foci colocalized with a centromere (compared with $16 \%$ before rotation), and $12 \%$ were located side-by-side (compared with $22 \%$ before rotation).

\section{Genotypes and staining}

To assess the frequency with which short Zip1 stretches are associated with centromeres, nuclei from a ZIP2-GFP@URA3 zip2::LEU2 CTF19-MYC-KAN strain were stained with antibodies to Zip1, GFP, and MYC. Zip1 stretches containing a single Zip2 focus were scored for association with Ctf19. To assess the frequency with which Zip1 stretches are flanked by telomeres, wild-type cells were stained with antibodies to Zip1 and Rap1 (Fig. 1B).

Centromere coupling and the localization of Ctf19 to axial associations were assessed by counting the number of centromere foci detected with anti-Red1 and anti-MYC antibodies. Ctf19 foci were counted in nuclei from a zip4::ADE2 CTF19MYC-KAN strain (Fig. 1C,E) and in nuclei from a zip1::LEU2 CTF19-MYC-KAN strain (Fig. 1D,F).

To assess colocalization between Zip3 and Ctf19, nuclei from ZIP3-GFP-URA3 CTF19-MYC-KAN strains were stained with antibodies to GFP and the MYC epitope (Fig. 2A-C). To assess colocalization between Zip2 and Ctf19, nuclei from a ZIP2GFP@URA3 zip2::LEU2 CTF19-MYC-KAN strain were stained with antibodies to Zip1, GFP, and MYC (Fig. 2D,E).

To examine the configuration of Zip2 relative to centromeres in short Zip1 stretches, early zygotene nuclei from a ZIP2. GFP@URA3 zip2::LEU2 CTF19-MYC-KAN strain were stained with antibodies to Zip1, GFP, and MYC (Figs. 3, 4). To examine the configuration of Zip3 relative to centromeres in short Zip1 stretches, early zygotene nuclei from a ZIP3-GFP-URA3 
CTF19-MYC-KAN strain were stained with antibodies to Zip1, GFP, and MYC (Fig. 4).

To assess the dependency requirements for Zip3 localization to centromeres (Fig. 5), spread nuclei from strains carrying ZIP3GFP-URA3 and CTF19-MYC-KAN were stained with antibodies to Red1, GFP, and MYC. To compare the frequencies with which Zip1 stretches are centromere associated in wild type and the zip3 mutant, nuclei from strains carrying CTF19-MYC-KAN were stained with antibodies to Zip1 and MYC.

\section{Acknowledgments}

We are grateful to Hideo Tsubouchi and Beth Rockmill for thoughtful discussions. This work was supported by the Howard Hughes Medical Institute.

\section{References}

Agarwal, S. and Roeder, G.S. 2000. Zip3 provides a link between recombination enzymes and synaptonemal complex proteins. Cell 102: 245-255.

Brown, P.W., Judis, L., Chan, E.R., Schwartz, S., Seftel, A., Thomas, A., and Hassold, T.J. 2005. Meiotic synapsis proceeds from a limited number of subtelomeric sites in the human male. Am. J. Hum. Genet. 77: 556-566.

Carpenter, A.T.C. 1988. Thoughts on recombination nodules, meiotic recombination, and chiasmata. In Genetic recombination (eds. R. Kucherlapati and G.R. Smith), pp. 529-548. American Society for Microbiology, Washington, D.C.

Cheng, C.-H., Lo, Y.-H., Liang, S.-S., Ti, S.-C., Lin, F.-M., Yeh, C.-H., Huang, H.-Y., and Wang, T.-F. 2006. SUMO modification control assembly of synaptonemal complex and polycomplex in meiosis of Saccharomyces cerevisiae. Genes \& Dev. 20: 2067-2081.

Chua, P.R. and Roeder, G.S. 1998. Zip2, a meiosis-specific protein required for the initiation of chromosome synapsis. Cell 93: 349-359.

Dong, H. and Roeder, G.S. 2000. Organization of the yeast Zip1 protein within the central region of the synaptonemal complex. J. Cell Biol. 148: 417-426.

Engebrecht, J. and Roeder, G.S. 1989. Yeast mer1 mutants display reduced levels of meiotic recombination. Genetics 121: 237-247.

Fung, J.C., Rockmill, B., Odell, M., and Roeder, G.S. 2004. Imposition of crossover interference through the nonrandom distribution of synapsis initiation complexes. Cell 116: 795802.

Henderson, K.A. and Keeney, S. 2005. Synaptonemal complex formation: Where does it start? Bioessays 27: 995-998.

Hooker, G.W. and Roeder, G.S. 2006. A role for SUMO in meiotic chromosome synapsis. Curr. Biol. 16: 1238-1243.

Joyce, E.F. and McKim, K.S. 2007. When specialized sites are important for synapsis and the distribution of crossovers. Bioessays 29: 217-226.

King, J.S. and Mortimer, R.K. 1990. A polymerization model of chiasma interference and corresponding computer simulation. Genetics 126: 1127-1138.

Lambie, E.J. and Roeder, G.S. 1986. Repression of meiotic crossing over by a centromere (CEN3) in Saccharomyces cerevisiae. Genetics 114: 769-789.

MacQueen, A.J., Phillips, C.M., Bhalla, N., Weiser, P., Villeneuve, A.M., and Dernburg, A.F. 2005. Chromosome sites play dual roles to establish homologous synapsis during meiosis in C. elegans. Cell 123: 1037-1050.
Novak, J.E., Ross-Macdonald, R.B., and Roeder, G.S. 2001. The budding yeast Msh4 protein functions in chromosome synapsis and the regulation of crossover distribution. Genetics 158: 1013-1025.

Page, S.L. and Hawley, R.S. 2004. The genetics and molecular biology of the synaptonemal complex. Annu. Rev. Cell Dev. Biol. 20: 525-558.

Pardo, B. and Marcand, S. 2005. Rap1 prevents telomere fusions by nonhomologous end joining. EMBO J. 24: 3117-3127.

Rockmill, B., Sym, M., Scherthan, H., and Roeder, G.S. 1995. Roles for two RecA homologs in promoting meiotic chromosome synapsis. Genes \& Dev. 9: 2684-2695.

Rockmill, B., Fung, J.C., Branda, S.S., and Roeder, G.S. 2003. The Sgs1 helicase regulates chromosome synapsis and meiotic crossing over. Curr. Biol. 13: 1954-1962.

Roeder, G.S. 1997. Meiotic chromosomes: It takes two to tango. Genes \& Dev. 11: 2600-2621.

Shinohara, M., Oh, S.D., Hunter, N., and Shinohara, A. 2008. Crossover assurance and crossover interference are distinctly regulated by the ZMM proteins during yeast meiosis Nat. Genet. 40: 299-309.

Smith, A.V. and Roeder, G.S. 1997. The yeast Red1 protein localizes to the cores of meiotic chromosomes. J. Cell Biol. 136: 957-967.

Sym, M. and Roeder, G.S. 1995. Zipl-induced changes in synaptonemal complex structure and polycomplex assembly. $J$. Cell Biol. 128: 455-466.

Sym, M., Engebrecht, J., and Roeder, G.S. 1993. Zip1 is a synaptonemal complex protein required for meiotic chromosome synapsis. Cell 72: 365-378.

Tsubouchi, T. and Roeder, G.S. 2005. A synaptonemal complex protein promotes homology-independent centromere coupling. Science 308: 870-873.

Tsubouchi, T., Zhao, H., and Roeder, G.S. 2006. The meiosisspecific Zip4 protein regulates crossover distribution by promoting synaptonemal complex formation together with Zip2. Dev. Cell 10: 809-819.

Zickler, D., Moreau, P.J.F., Huynh, A.D., and Slezec, A. 1992. Correlation between pairing sites, recombination nodules and meiotic recombination in Sordaria macrospora. Genetics 132: 135-148. 


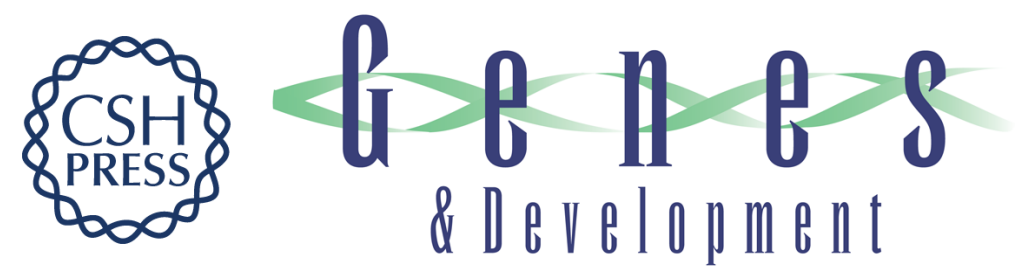

\section{Initiation of meiotic chromosome synapsis at centromeres in budding yeast}

Tomomi Tsubouchi, Amy J. MacQueen and G. Shirleen Roeder

Genes Dev. 2008, 22:

Access the most recent version at doi:10.1101/gad.1709408

References This article cites 26 articles, 12 of which can be accessed free at: http://genesdev.cshlp.org/content/22/22/3217.full.html\#ref-list-1

License Freely available online through the Genes \& Development Open Access option.

Email Alerting Receive free email alerts when new articles cite this article - sign up in the box at the top Service right corner of the article or click here.

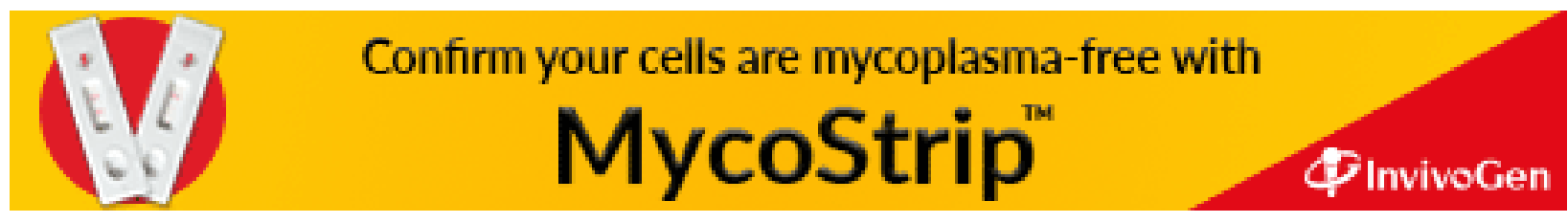

